\title{
A Comparitive Study of Data Forwarding Schemes in VANET
}

\author{
Ms. V. Bhuvaneshwari ${ }^{1}$, Dr. S.J.K. Jagadeesh Kumar ${ }^{1}$, Ms. V.R. Azhaguramyaa ${ }^{1}$ \\ Department of Computer Science and Engg, Sri Krishna College of Engineering and Technology, Coimbatore, India ${ }^{1}$
}

\begin{abstract}
VANET is a promising communication technology that enables communication among V2V and V2I for improving driving safety and efficiency. In VANET, data transfer is done with the help of multi-hop communication in which vehicles act as data carrier. Intelligent Transportations System (ITS) has deployed a no of RSU (Road Side Units) along the roads to collect and deliver traffic information from TCC (Traffic Control Centre) to the vehicles. This paper compares six vehicle trajectory-based data forwarding schemes, tailored for vehicular ad hoc networks. Nowadays GPS-based navigation systems are used for providing efficient driving paths for drivers. Subsequently it also discusses the performance of those data forwarding schemes and list the merits and demerits and conclude the challenges facing in the present day research in Vehicular ad hoc Network (VANET).
\end{abstract}

Keywords: VANET, IVC, ITS, RSU, Vehicle trajectory, VADD, TBD, TSF, TOAF, STDFS, SDFM.

\section{INTRODUCTION}

Recently, it has been widely accepted by the academic society and industrial corporation that the cooperation between vehicles and road transportation systems can significantly improve driver's safety and road efficiency. Vehicular Ad-hoc NETwork (VANET) is a subgroup of Mobile Ad-hoc network (MANET), where communicating nodes are replaced by moving vehicles. It is an important component of ITS (Intelligent Transport System). In VANETs, [1] vehicles can able to communicate each other (V2V, Vehicle-to-Vehicle communications) also they can connect to an infrastructure (V2I, Vehicle-toInfrastructure) to get some service like accident alerts, traffic alerts, road condition and weather information. This infrastructure called Road Side Units (RSU) is located along the roads to ensure service coverage. Nodes in VANETs are highly mobile, thus the network topology is ever-changing. regulations. In general, VANETs [1] have normally higher computational capability and higher transmission power than MANETs. The vehicular communication [5] for the driving safety and efficiency has been feasible through the standardization of Dedicated Short Range Communications (DSRC) as IEEE $802.11 \mathrm{p}$ in 2010. As an important trend for the vehicular based networking, Global Positioning System GPS-based navigation systems [5] are popularly used by drivers.

Fig. 1 shows the components and communications with a typical VANET. In a typical VANET, Vehicles communicate with each other through V2V communication in Ad hoc network fashion, and V2I communication through road-side-units (RSU) and mobile broadband (e.g. 4G/LTE). OBUs (On Board Units) of various vehicles form a mobile ad hoc network (MANET). OBUs and road side units together will form ad-hoc network. An RSU can be attached to an infrastructure network, which in turn can be connected to the Internet.

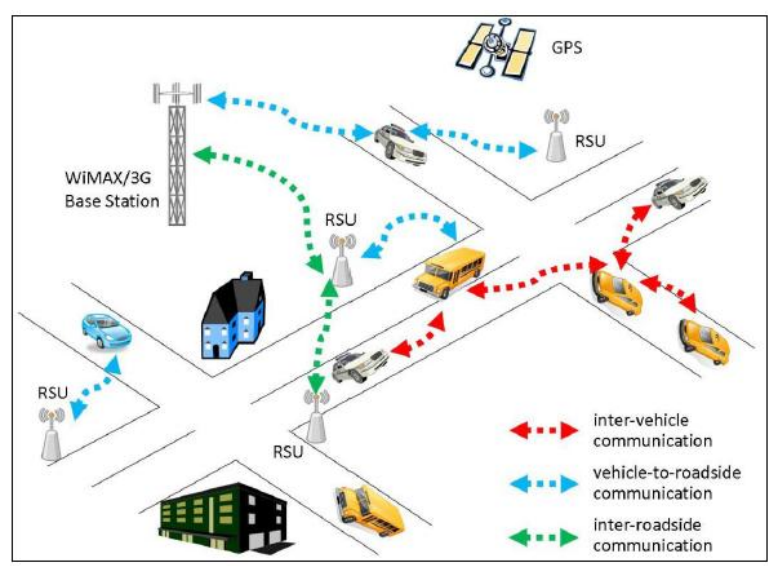

Fig 1: Architecture of VANET

This paper is structured as follows. The summary of the related work of vehicular networking is elaborated in section II. This is followed by a detailed description of vehicular traffic statistics and vehicular trajectory based six data forwarding schemes in section III. Then the comparative analysis of six data forwarding schemes is provided in section IV. Section V concludes with suggesting the extension of proposed work.

\section{RELATED WORK}

Most of the data forwarding schemes in VANET are designed aiming at building a path with shorter delivery delay from a source to a destination vehicle. Vahdat et al. and Becker et al. proposed the idea of the carry-and forward which is used in dealing with frequent network partitioning and merging. Packets can be forwarded if there are nodes nearby. However, this protocol is not specifically designed for MANET and ignored the fact that the trajectory of a moving vehicle. In order to modify the 
model and let it become suitable for VANET, Zhao et al. and $\mathrm{CaO}$ et al. proposed a data forwarding scheme and constructs a link delay model called vehicle carry-andforward model (VADD [4]). TSF is a trajectory based data forwarding scheme to select a RSU as the target point in VANET, which is proposed by Jeong et al. In [2] Xu et al. designed a shared trajectory based forwarding scheme for V2V transmission [4] which used the predicted encounter graph to minimize the delivery delay. However, these trajectory based data forwarding schemes are hard to be realized in the real-world since people may not want to share their own trajectories considering the privacy issue. The concept of using public transportation for data delivery has been considered nowadays. Wong et al. proposed an architecture of BUSNet. They attempted to take advantage of public transportation with predictable routes for improving the inter-vehicle communications.

\section{DATA FORWARDING SCHEMES}

Data forwarding is the relaying of packets from one network segment to another network segment or from source to destination by nodes in a computer network. The Network Layer of the OSI Layer is responsible for Packet Forwarding. In this section, we describe five data forwarding schemes, such as VADD [4], TBD [2], TSF [3], TOAF[8], STDFS [9] and SDFM[7].

\section{VADD: Vehicle-Assisted Data Delivery for V2I}

\section{Data Delivery}

VADD [4] is a data forwarding scheme for the V2I data delivery, based on vehicular traffic statistics, such as the vehicle arrival rate and average speed per road segment along with the digital roadmaps provided by GPS based navigation systems [15]. For example, as shown in Fig. 2 [5], the current packet carrier (denoted as Carrier) wants to deliver its packet to AP (Access Point) in the road network. It has two neighboring vehicles [10] (denoted as car1 and car2) within its communication range.

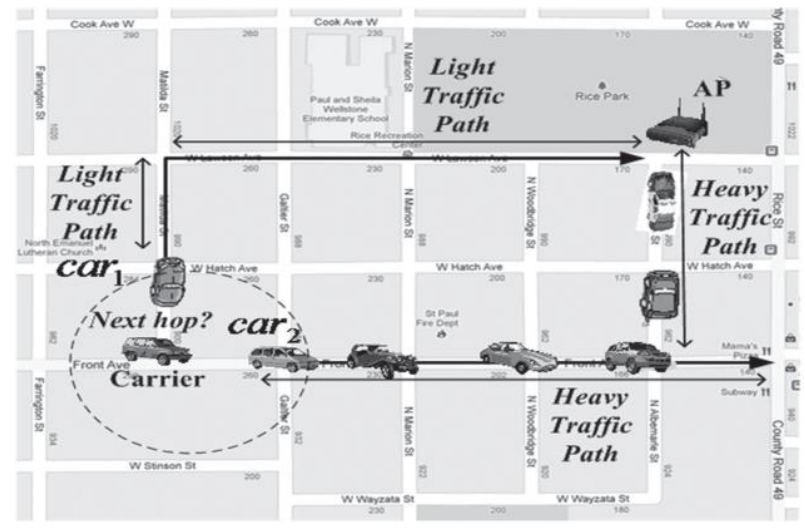

Fig. 2. [5] VADD Data Forwarding in Road Network

carl's trajectory passes through a light traffic path where a few vehicles are moving in that path. On the other hand, car2's trajectory [10] passes through a heavy traffic path where a lot of vehicles are moving statistically. In this case, definitely, Carrier [5] needs to forward its packets to car2 as a next-hop carrier rather than car1. Even though VADD [5] solves the data forwarding issue nicely through the linear systems of recursive equations, the limitation of VADD [5] does not use the vehicle trajectory available for a better forwarding metric computation.

\section{TBD: Trajectory-Based Data Forwarding for} V2I Data Delivery

TBD [2] is a data forwarding scheme to improve VADD for the V2I(Vehicle-to-infrastructure) data delivery, using not only vehicular traffic statistics, but also vehicle trajectory in the privacy-preserving manner.

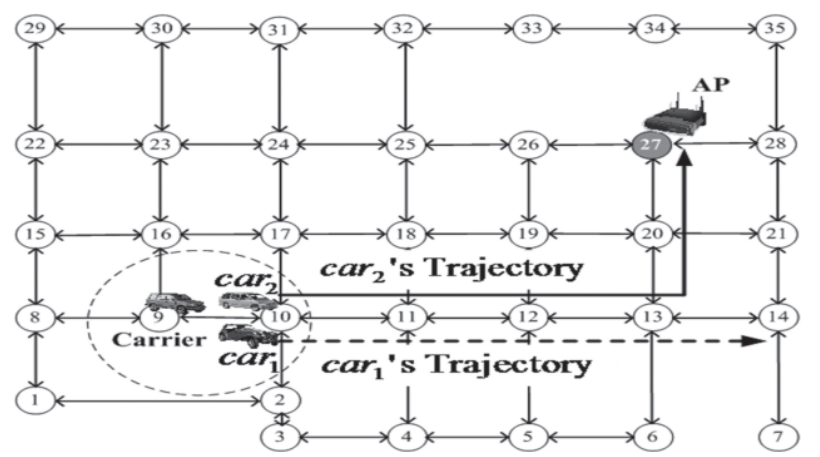

Fig3 TBD Data Forwarding in Road Network

In Fig. 3 [5], the current packet carrier [5] (denoted as Carrier) has only two neighboring vehicles (denoted as car1 and car2) for the next-hop carrier to forward the data in this road network. The next-hop carrier candidates [10] car 1 and car2 are moving at the same coordinate and in the same direction towards intersection 11. One difference is that carl's trajectory is far away from the communication range [10] with AP (Access Point) and car2's trajectory passes through AP. In this case, car2 should be selected by Carrier as a next-hop carrier to forward the data because car2 has a high chance to deliver Carrier's packets to AP. Therefore, TBD can allow individual vehicles to calculate their own EDD [10] based on their own trajectory so that the packet carrier can select the best next-hop carrier among its neighboring vehicles to forward the data.

\section{TSF: Trajectory-Based Statistical Forwarding for I2V Data Delivery}

TSF [2] is a data forwarding scheme for the infrastructureto-vehicle $(\mathrm{I} 2 \mathrm{~V})$ data delivery, using the trajectory of the destination vehicle. TSF [3] forwards the packets over multi-hop to a selected target point (AP) where the vehicle is expected to pass by. Such a target point (AP) [10] is selected optimally to minimize the packet delivery delay while satisfying the required packet delivery probability.

Once the target point [3] is decided or identified, TSF adopts the source routing technique, i.e., forwards the packet using a shortest-delay forwarding path specified by multiple intersections in the target road network. Fig. 4 [5] shows the I2V (Infrastucture-to-Vehicle) data delivery from AP1 to Destination Vehicle. 
Vol. 5, Issue 12, December 2016

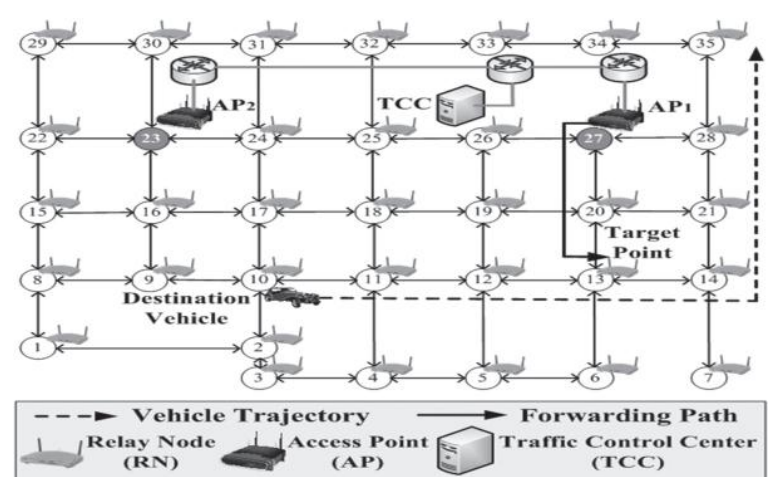

Fig. 4. TSF Data Forwarding in Road Network

In the figure 4 [10], AP1 selects intersection 13 (denoted as n13) as a target point through the current position and trajectory info., of Destination Vehicle; note that the current positions and trajectories of vehicles are available to APs via Traffic Control Center (TCC) [18] because the vehicles peroidically update their current position and trajectory in TCC. One limitation of TSF [5] is to require relay nodes as infrastructure nodes ie., Access Points or RSU for the reliable I2V data delivery.

\section{TOAF: Trajectory-Based Optimal Area} Forwarding for I2V Data Delivery

Trajectory-based optimal area forwarding (TOAF)[8] scheme is data forwarding scheme for the multihop (I2V) data delivery with partial deployment of stationary nodes(Acess Points). In general, many intersections of real world do not have to contain stationary nodes, and some stationary nodes[8] may be located on roadsides rather than at intersections in road.

8

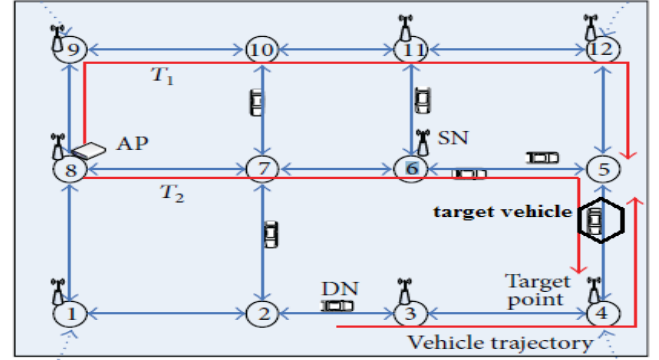

(8)

Fig. 5. TOAF Data Forwarding in Road Network

In figure 5 [8] ,If AP predicts that selecting a stationary node in the trajectory of the destination vehicle in TTL(Time To Live) is impossible, then AP selects a target vehicle as relay node from vehicles that could encounter the destination vehicle .

\section{STDFS: Shared-Trajectory-Based Data Forwarding} Scheme for V2V Data Delivery

STDFS [10] is a data forwarding scheme for the multihop vehicle-to-vehicle (V2V) data delivery through the sharing of the vehicular trajectories moving in a target road network. The privacy-sensitive users can opt out, while participatory users can exchange privacy information for convenience and performance. A predicted encounter graph[9] is constructed to find the next hop. Based on the predicted encounter graph, STDFS optimizes the forwarding sequence to achieve the minimal delivery delay given a delivery ratio threshold[9]. Fig. 6 [5] shows the data forwarding from vehicle a (denoted as $\mathrm{Va}$ ) to stationary vehicle $\mathrm{s}$ (denoted as Vs ) via the intermediate vehicles $\mathrm{b}$ or $\mathrm{d}$ (denoted as $\mathrm{Vb}$ or $\mathrm{Vd}$ ).

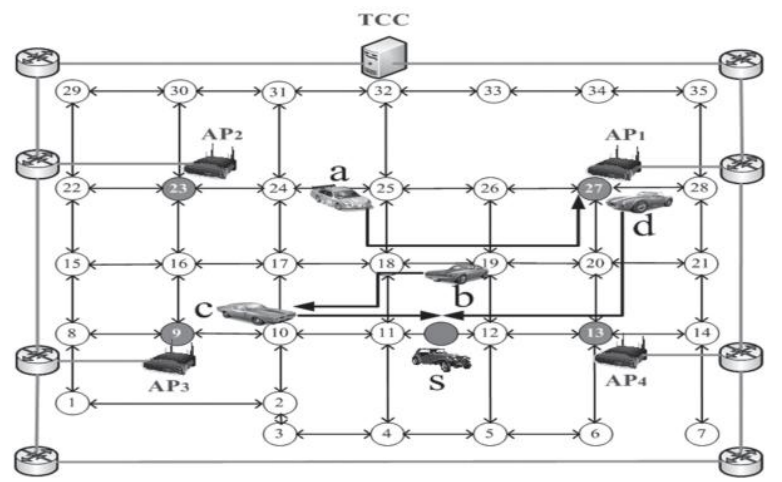

Fig.6. STDFS Data Forwarding in Road Network

In STDFS, source vehicle constructs the predicted encounter graph to determine the next-hop carrier to forward the data that can guarantee the user-defined delivery probability like in TSF[5]. In Fig. 6 [5], Va is the source vehicle and Vs is the destination vehicle. The vehicles $\mathrm{Vb}, \mathrm{Vc}$ and $\mathrm{Vd}$ are intermediate carriers. $\mathrm{Vb}$ and $\mathrm{Vd}$ may be the next encountered vehicles for Va for data forwarding[5]. In the expansion of the predicted encounter graph, $\mathrm{Vb}$ may encounter $\mathrm{Vc}$ and then $\mathrm{Vc}$ may finally encounter the destination vehicle Vs[10] .In the same way, $\mathrm{Vd}$ may finally encounter the destination vehicle Vs. The neighboring vehicles individually calculate their own EDR(Expected Delivery Ratio)[5] and EDD(Expected Delivery Delay) for the selection of next-hop carrier.

\section{SDFM: A Social-based Data Forwarding Mechanism for V2V Communication in VANETs}

SDFM (A Social-based Data Forwarding Mechanism) is a data forwarding scheme to improve STDFS for the multihop vehicle-to-vehicle data delivery through storecarry-forward method. The SDFM[7] learns social characteristics of vehicles in a distributed manner, and then transfers messages in a "Store-Carry-Forward" method.

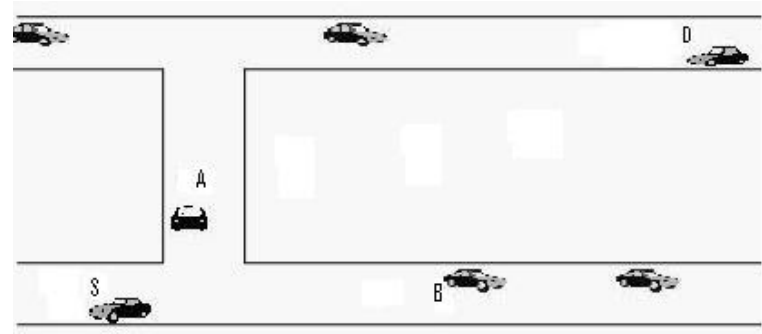

Fig.7. SDFM Data Forwarding in Road Network 
Vol. 5, Issue 12, December 2016

SDFM describes the sociality of a vehicle as centrality and TBD has a shorter delivery delay than VADD from the community. Based on community and centrality[7], a lowest vehicular density to the highest vehicular density vehicle forwards it to the vehicle with higher global by a more effective delivery delay estimation using the centrality than itself till it meets a vehicle within the same individual vehicle trajectory.

community as the message's final destination, and then forwards the data packet to the vehicle with higher local centrality till it is finally delivered[7]. In SDFM[7], vehicles periodically records meeting histories with other vehicles, as well as their own moving trajectories in a certain time window. The optimal way of assessing centrality is to count how many times the node is on the shortest forwarding paths for all the traffic flows in the vehicular network.

\section{THE ANALYSIS OF FORWARDING SCHEMES}

In this section, the Six forwarding schemes (i.e., VADD, TBD, TSF, TOAF and STDFS,SDFM) are analyzed based on the forwarding type that are explained in Section III. Table 4 shows the comparison among those schemes. Fig. 8 shows the performance of VANET data forwarding schemes.

A. FORWARDING TYPE-V2I: The two data forwarding schemes that can support the forwarding type Vehicle-to-Infrastructure are VADD and TBD.

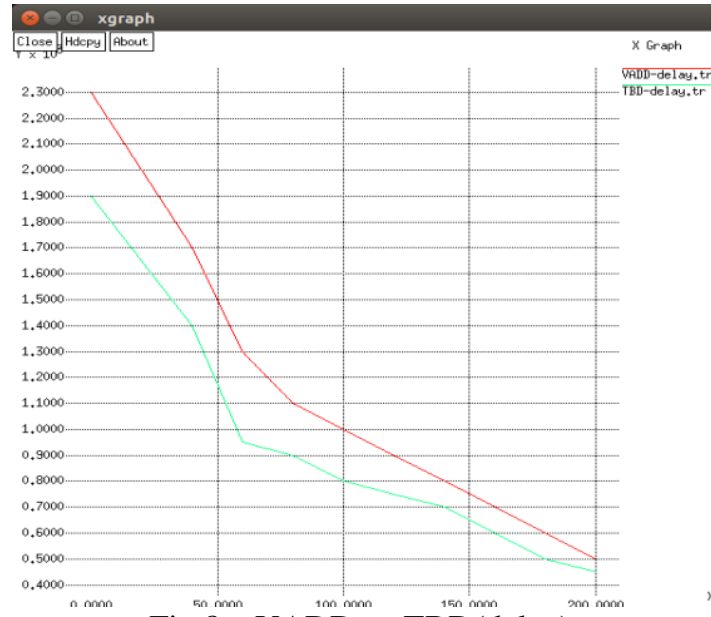

Fig 8a: VADD vs TBD(delay)

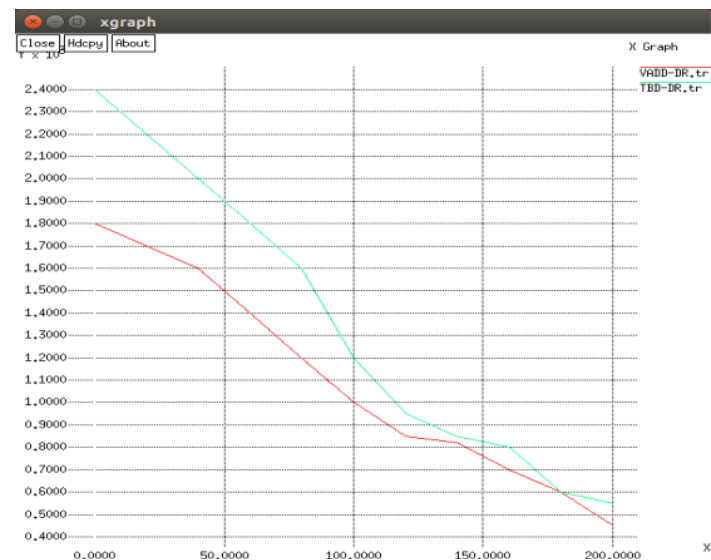

Fig 8b: VADD vs TBD delivery rate
Table 1: The Comparison between VADD and TBD Forwarding Schemes for Vehicular Ad Hoc Networks

\begin{tabular}{|l|l|l|}
\hline \multicolumn{3}{|c|}{ NO OF VEHICLES(50) } \\
\hline METHOD & DELIVERY RATE(\%) & DELIVERY DELAY(MS) \\
\hline VADD & $70 \%$ & $150 \mathrm{~ms}$ \\
\hline TBD & & \\
\hline
\end{tabular}

B. FORWARDING TYPE-I2V: The two data forwarding schemes that can support the forwarding type Infrastructure-to-vehicle are TSF and TOAF. TSF uses an intermediate infrastructure node called target point to forward the packet to the moving destination vehicle. Whereas TOAF uses an intermediate vehicle as a target point to forward data to the moving destination vehicle.

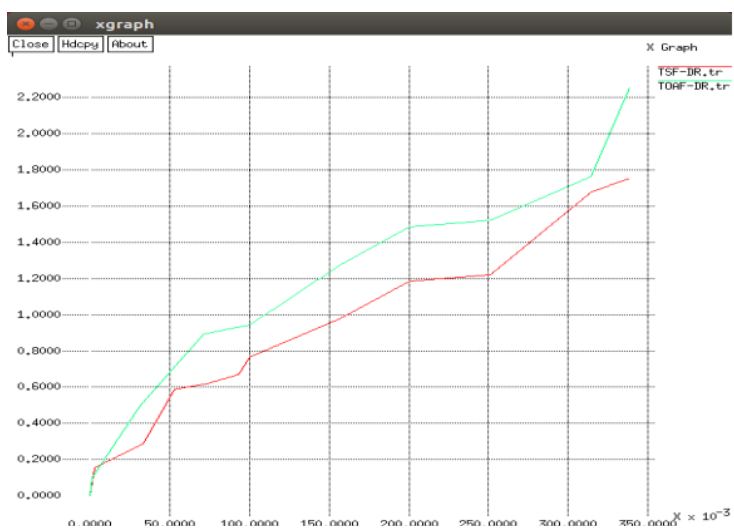

Fig 8c: TSF vs TOAF - delivery rate

As shown in Figure 8c, the delivery delay in TSF, TOAF decrease as the number of vehicles increases. This is because the more vehicles increase the forwarding probability among vehicles, so this reduces the carry delay, leading to the overall shorter delivery delay.

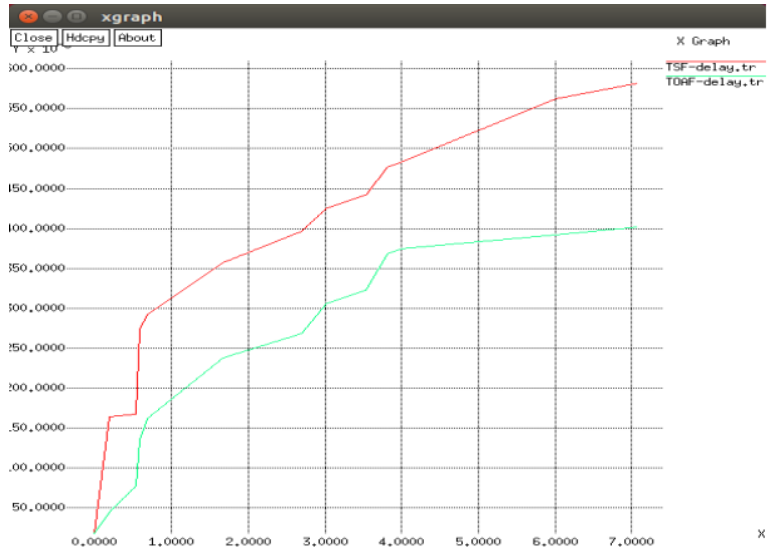

Fig 8d: TSF vs TOAF (delay) 
Vol. 5, Issue 12, December 2016

Table 2: The Comparison between TSF and TOAF Forwarding Schemes for Vehicular Ad Hoc Networks

\begin{tabular}{|l|l|l|}
\hline \multicolumn{3}{|c|}{ NO OF VEHICLES(50) } \\
\hline METHOD & DELIVERY RATE(\%) & DELIVERY DELAY(MS) \\
\hline TSF & $85 \%$ & $100 \mathrm{~ms}$ \\
\hline TOAF & $90 \%$ & $85 \mathrm{~ms}$ \\
\hline
\end{tabular}

C. FORWARDING TYPE-V2V: The two data forwarding schemes that can support the forwarding type V2V data delivey are STDFS and SDFM. STDFS uses predicted encounter graph and shortest path algorithm to find next hop to forward the data. Whereas SDFM uses community and centrality information of a vehicle to forward the data.

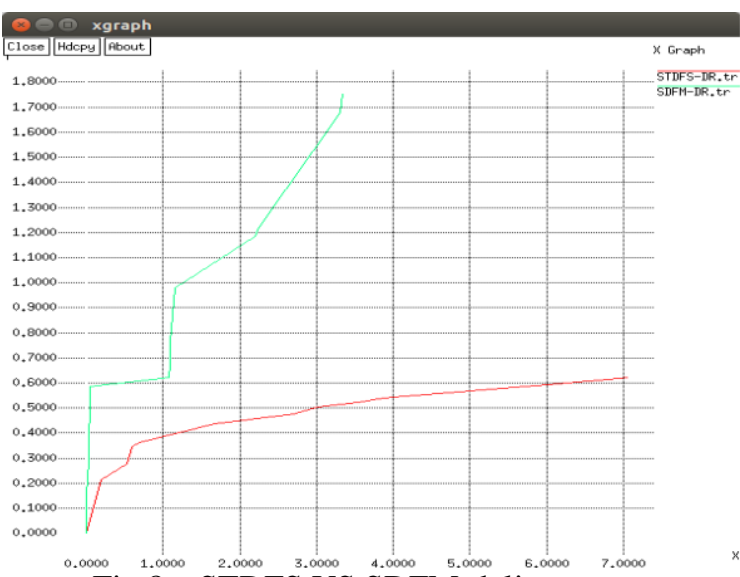

Fig 8e: STDFS VS SDFM-delivery rate

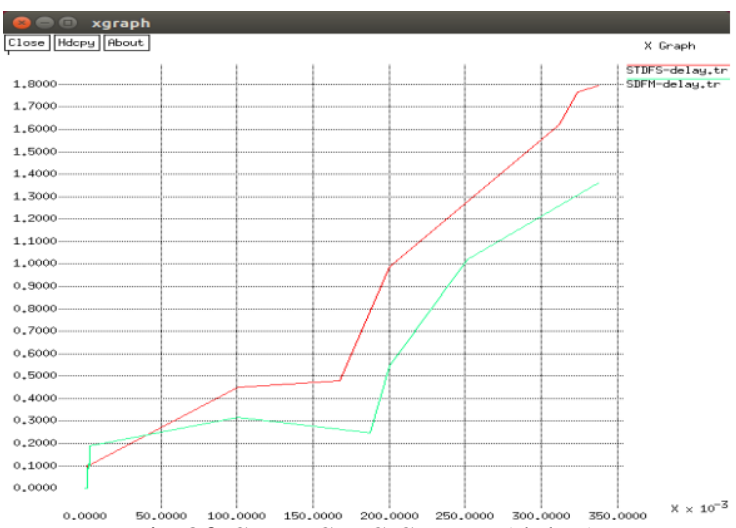

Fig 8f: STDFS VS SDFM (delay)

Table 3: The Comparison between STDFS and SDFM Forwarding Schemes for Vehicular Ad Hoc Networks

\begin{tabular}{|l|l|l|}
\hline \multicolumn{3}{|c|}{ NO OF VEHICLES(50) } \\
\hline METHOD & DELIVERY RATE(\%) & DELIVERY DELAY(MS) \\
\hline STDFS & $91 \%$ & $98 \mathrm{~ms}$ \\
\hline SDFM & $95 \%$ & $70 \mathrm{~ms}$ \\
\hline
\end{tabular}

The community and centrality information provides more detailed knowledge than vehicular statistics, SDFM could forward packets through better paths, and it is more suitable for data forwarding when vehicular networks become sparse.

Table 4: The Comparison among six Data Forwarding Schemes for Vehicular Ad Hoc Networks

\begin{tabular}{|c|c|c|c|c|c|}
\hline $\begin{array}{l}\text { FORWARDNG } \\
\text { SCHEME }\end{array}$ & \begin{tabular}{|l|} 
FORWARDNG \\
TYPE
\end{tabular} & $\begin{array}{l}\text { VEHICUIAR } \\
\text { STATISTICS }\end{array}$ & $\begin{array}{l}\text { VEHICE } \\
\text { TRAJECTOEY }\end{array}$ & $\begin{array}{l}\text { INFRASTRUCTUKE } \\
\text { NODES }\end{array}$ & $\begin{array}{l}\text { PRIVACY } \\
\text { EXPOSURE }\end{array}$ \\
\hline VADD & v21 & YES & NO & ACCESS POINTS & NO \\
\hline TBD & v21 & YES & YES & ACCESS POINTS & NO \\
\hline TSF & $12 \mathrm{~V}$ & YES & YES & $\begin{array}{l}\text { ACCEESS POINTS } \\
\text { RELAY NODES,TCC }\end{array}$ & NO \\
\hline TOAF & $\mathrm{I} 2 \mathrm{~V}$ & YES & YES & $\begin{array}{l}\text { ACCESS POINTS } \\
\text { RELAYNODES(VEH } \\
\text { ICLE),TCC }\end{array}$ & NO \\
\hline STSFS & $\mathrm{V}_{2} \mathrm{~V}$ & YES & YES & $\begin{array}{l}\text { ACCESS POINTS, } \\
\text { TCC }\end{array}$ & YES \\
\hline SDFM & V2V & YES & YES & $\begin{array}{l}\text { ACCESS POINTS, } \\
\text { TCC }\end{array}$ & NO \\
\hline
\end{tabular}

All of six forwarding schemes use vehicular traffic statistics for their forwarding metric computation[10]. Except for VADD, the remaining five schemes take advantage of vehicle trajectory for the more efficient forwarding metric computation

\section{CONCLUSION}

Data forwarding is one of the important parameter in vehicular communication. Thus this paper explained six data forwarding schemes based on vehicle trajectory in vehicular networks except VADD which is based only on vehicular traffic statistics. The vehicle trajectory is a useful property in the design of data forwarding schemes because it allows for either a better forwarding metric computation or a better destination location estimation . As future work, we will investigate more the characteristics of vehicular trajectory to make better data forwarding schemes that can work well in all the three data forwarding types $\mathrm{V} 2 \mathrm{~V}, \mathrm{~V} 2 \mathrm{I}, \mathrm{I} 2 \mathrm{~V}$, considering the minimization of delivery delay, the privacy protection on trajectory.

\section{REFERENCES}

[1] Bhuvaneshwari.S, Divya.G, Kirithika.K.B and Nithya.S," A SURVEY ON VANET", International Journal of Advanced Research in Electrical, Electronics and Instrumentation Engineering Vol. 2, Issue 10, October 2013.

[2] J. Jeong, S. Guo, Y. Gu, T. He, and D. Du, "Trajectory-Based Data Forwarding for Light-Traffic Vehicular Ad Hoc Networks," IEEE Transactions on Parallel and Distributed Systems, vol. 22, no. 5, pp. 743-757, May 2011.

[3] J. Jeong, S. Guo, Y. Gu, T. He, and D. Du, "Trajectory-based Statistical Forwarding for Infrastructure-to-Vehicle Data Delivery in vehicular network," IEEE Transactions on Mobile Computing (TMC), March 2010.

[4] J. Zhao and G. Cao, "VADD: Vehicle-Assisted Data Delivery in Vehicular Ad Hoc Networks," IEEE Transactions on Vehicular Technology, vol. 57, no. 3, pp. 1910-1922, May 2008. 
[5] Jaehoon (Paul) Jeong, Department of Software, Sungkyunkwan University, Suwon, Republic of Korea" Vehicle Trajectory-Based Data Forwarding Schemes for Vehicular Ad Hoc Networks",2012

[6] K.-J. Wong, B. S. Lee, B. C. Seet, G. Liu, and L. Zhu, "BUSNet: Model and usage of regular traffic patterns in mobile ad hoc networks for inter-vehicular communications," in Proc. Patterns Mobile Ad Hoc Networks Inter Vehicular Communications, 2003.

[7] Rui Tian, Zhenzhen Jiao, Guiyun Bian, Zhiqing Huang, Yibin Hou," A Social-based Data Forwarding Mechanism for V2V Communication in VANETs", 2015

[8] Liang-Yin Chen,1 Song-Tao Fu,1 Jing-Yu Zhang, 1 Xun Zou,1 Yan Liu,2 and Feng Yin3," Trajectory-Based Optimal Area Forwarding for Infrastructure-to-Vehicle Data Delivery with Partial Deployment of Stationary Nodes(TOAF)", International Journal of Distributed Sensor Networks, March 2013.

[9] F. Xu, S. Guo, J. Jeong, Y. Gu, Q. Cao, M. Liu, and T. He, "Utilizing shared vehicle trajectories for data forwarding in vehicular networks," in Proc. IEEE INFOCOM, Apr. 2011, pp. 441-445.

[10] Jaehoon (Paul) Jeong1, Tian He2, and David H. C. Du2," Trajectory Based Data Forwarding Schemes for Vehicular Networks", ZTE COMMUNICATIONS,2014.

[11] A. Soua and H. Afifi, "Adaptive data collection protocol using reinforcement learning for VANETs," in Proc. Int. Wireless Communications Mobile Computing Conf., July 2013, pp. 10401045.

[12] Xiaoxiao jiang and David H.C.Du,"Bus-Vanet with traffic infrastructure" in IEEE ITS, April 2015.

[13] Jing Zhao, "VADD IN VANET", in IEEE ,May 2008.

[14] Feliz Kristianto karnadi , Zhi Hai Mo and Kun-chan Lan ,"Rapid Generation of realistic mobility models for VANET",2010.

[15] Fulong Xu, Shuo Guo,Qing Cao ,"Utilizing STDFS in VANET",IEEE 2011

[16] Sandeep Kumar, Kantveer, Vehicle Assisted Data Delievery Technique To Control Data Dissemination In (Vanets) , 2015

[17] Xiaoxiao Jiang and David H.C. Du,Department of Computer Science and Engineering, University of Minnesota, USA,"BUSVANET integrated with traffic infrastructure",2015.

\section{BIOGRAPHY}

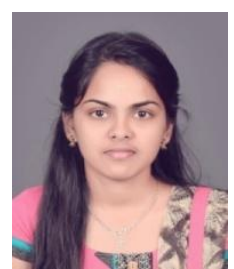

Bhuvaneshwari $\mathbf{V}$ received a $\mathrm{BE}$ degree in Computer Science and Engineering from Karpagam college of Engineering in 2014. She currently purses ME in the Department of Computer Science and Engineering at the Sri Krishna College of Engineering and Technology, Coimbatore, India. Her research interests include vehicular ad-hoc networks, Mobile ad-hoc networks. 